\title{
L-arginine delivery based on peptide-like polymeric molecules contributes to changes in nitric oxide biosynthesis and vascular
}

tone

\author{
Dmitry Yaroslavtsev ${ }^{1}$, Anastasiya Solovieva ${ }^{1}$, Anastasiya Kotlyarova ${ }^{1, *}$, German Baykalov ${ }^{1,2}$, \\ Konstantin Ershov ${ }^{2}$, Pavel Madonovi,2 \\ ${ }^{1}$ Researcher Institute of Clinical and Experimental Lymphology- Branch of the Institute of Cytology and Genetics Siberian Branch of the \\ Russian Academy of sciences, Novosibirsk, Russian Federation \\ ${ }^{2}$ Novosibirsk State Medical University, Novosibirsk, Russian Federation
}

\section{Abstract}

Peptides containing arginine and capable of delivering it into cells can be considered as a source of nitric oxide synthase (NOS) substrate for increasing NO synthesis. NO, in turn, is a key antithrombotic and antiatherosclerotic factor, as it dilates blood vessels, inhibits platelet function, prevents leukocytes from adhering to vascular walls, and restricts smooth muscle vascular proliferation. Thus, preparations based on biodegradable arginine-containing polymers are promising direction in the treatment of hemocirculation disorders. Here we showed synthetic approach to make new peptide-like biodegradable polymer with specific metabolic-related properties. Its ability to increase NO production in vitro and vasodilator action in vivo was evaluated. The backbone structure of the proposed polymer is a sequence of cysteines polymerized into linear or cyclic molecules through disulfide bridges and diamine spacers; L-arginine residues are grafted onto the main chain via peptide bonds. Consequently, the structure bears a set of properties of cell penetrating peptides (CPP), which provides the ability to move across the cell membrane and indirectly increase the bioavailability of arginine. The proposed polymeric molecules are stable in oxidative media, but could be easily depolymerized in a reducing intracellular environment with the formation of thiol-containing monomers, prone to intracellular enzymatic hydrolysis yielding amino acids (arginine and cysteine). That sort of Redox degradation significantly eliminates the potential danger of the toxic effect of the original polymer (a kind of polycation in fact). In vitro experiments have shown significant increase in NO production compared to octa-arginine (reference) and monomeric arginine (control). In vivo study has shown significant decrease in blood pressure compared to intact controls. As a result, a peptide-like L-arginine grafted polymeric structure demonstrating CPP-properties can be considered a promising compound for creating vasoactive drugs

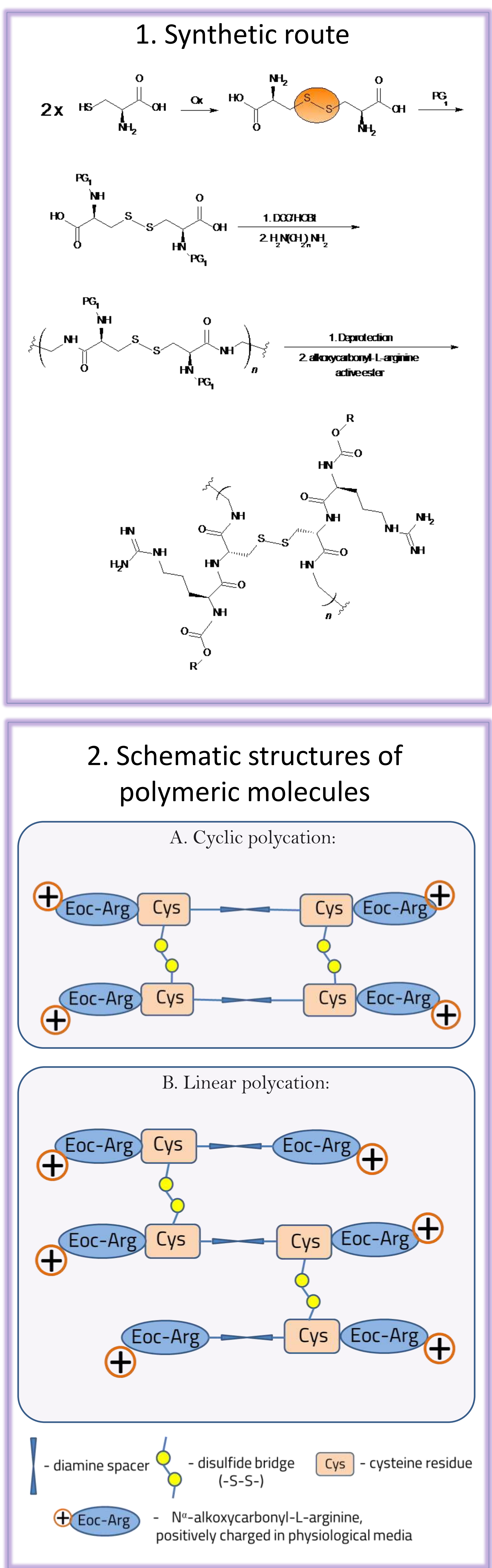

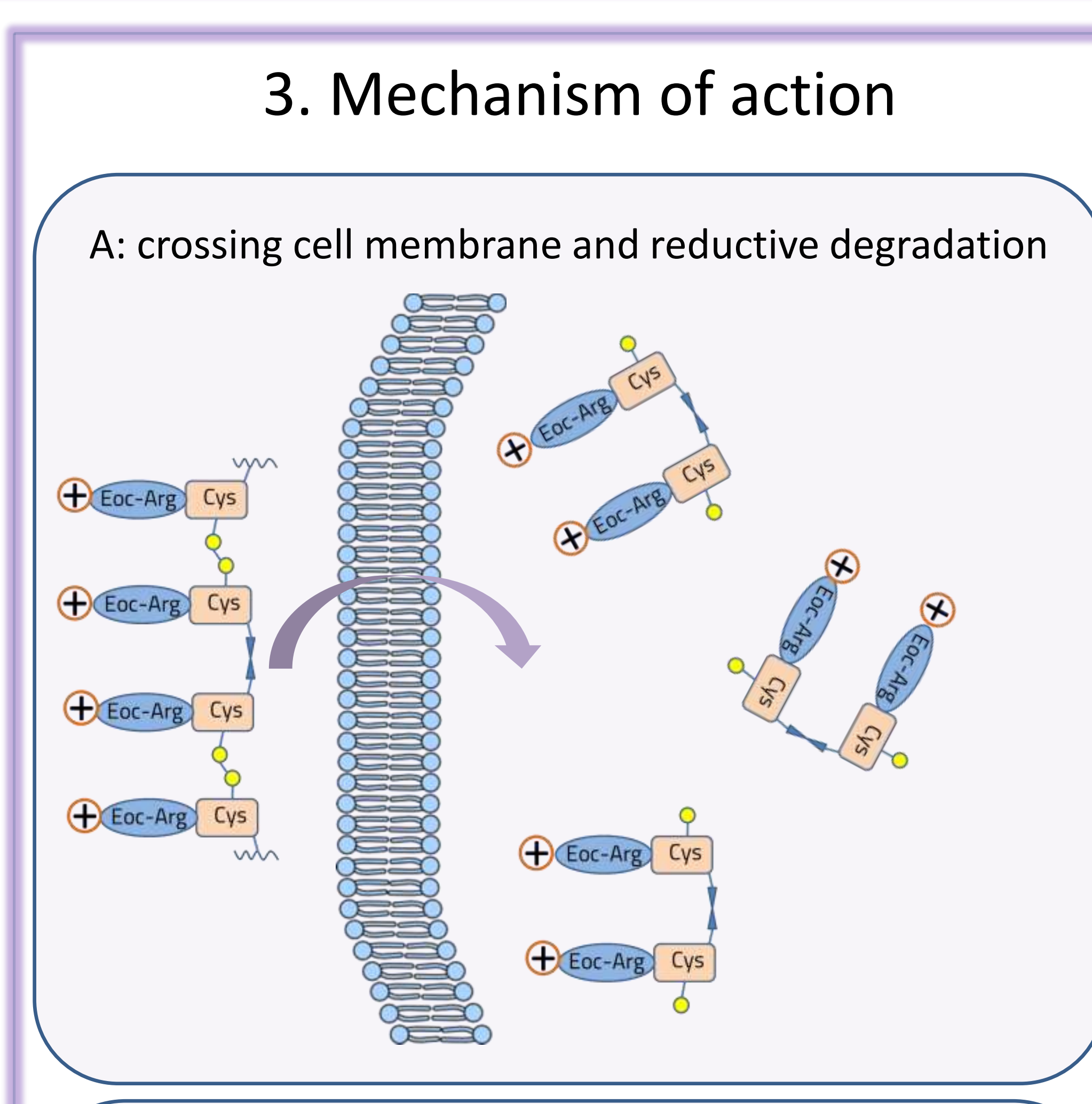

B: hydrolysis of peptide and amide bonds

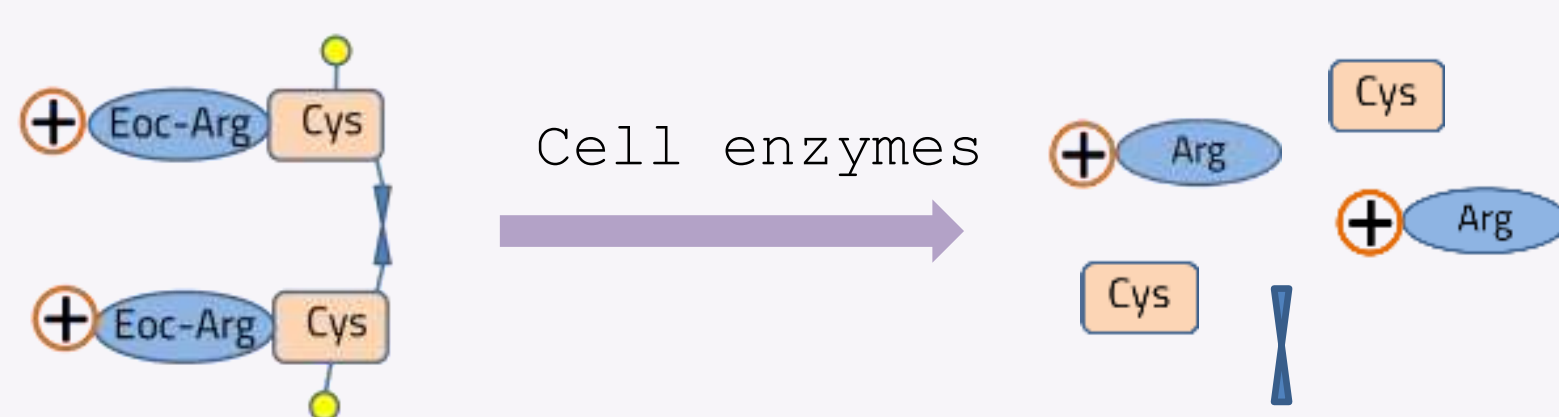

$$
\text { C: NOS-dependent nitric oxide generation }
$$

$$
\begin{aligned}
& \oplus_{\text {ARB }} \rightarrow \text { AB NO-Synthase }(\text { No. NO. } \\
& \oplus
\end{aligned}
$$

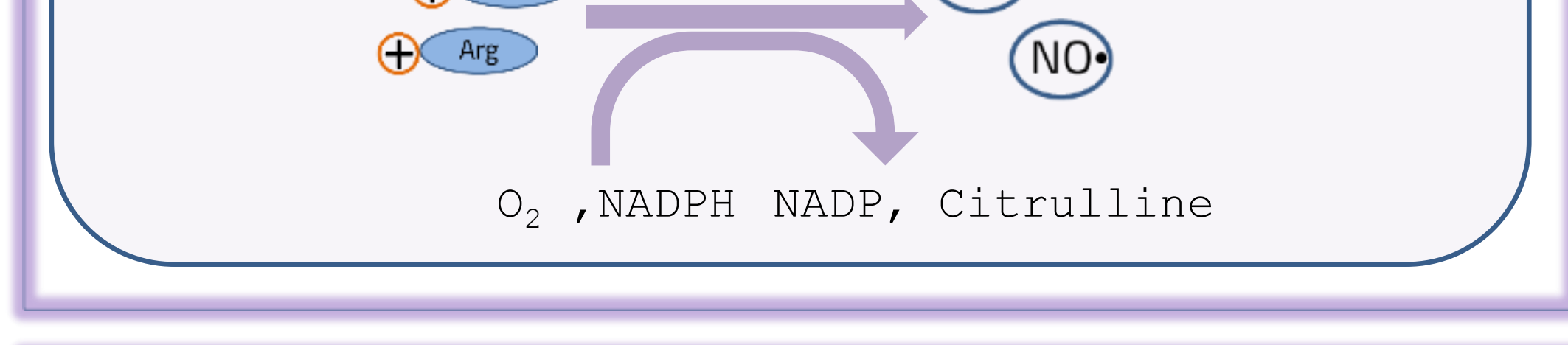

4. Molecule summary

Properties and advantages of the structure:

- Moderate molecular weight ( $<5 \mathrm{kDa})$

- Redox sensitive: stable in oxidizing media

White, non-hygroscopic solid

Monomeric blocks are non-toxic: L-amino acids and diamines

It is bearing a set of properties of cell penetrating peptides (CPP)

May be modified to carry different cargoes

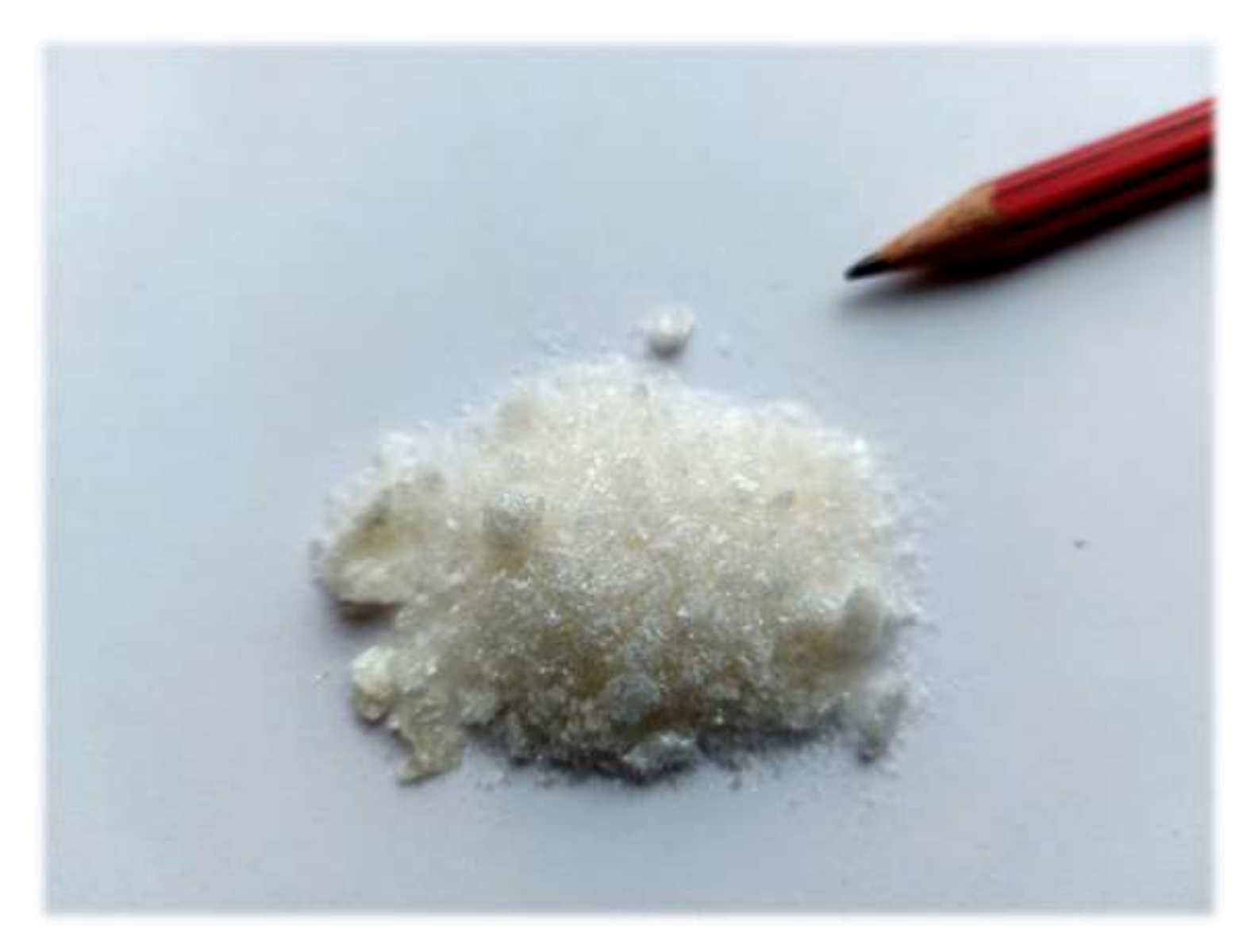

5. In vitro and in vivo experiments to determine the pharmacological activity

A: Dynamics of NO formation in endothelial cells after polymer exposure [C] NO2-, $\mu \mathrm{mol}$

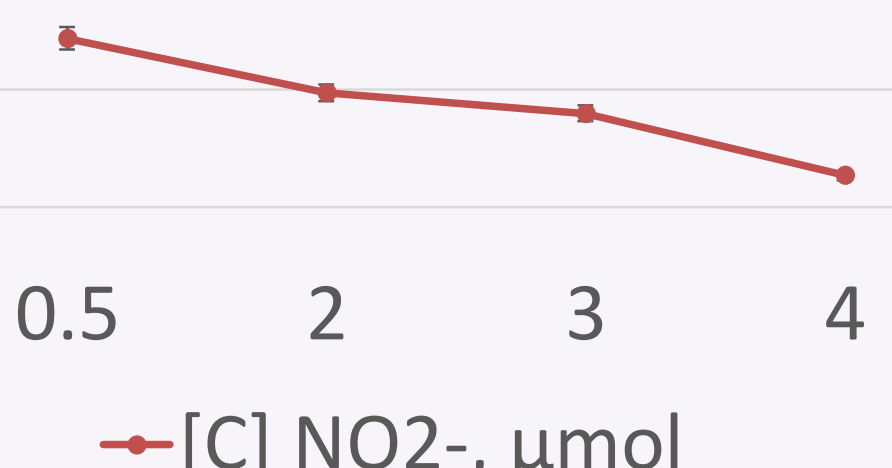

$\rightarrow[\mathrm{C}] \mathrm{NO2}-$, umol

It was determined that after 30 minutes the maximum amount of $\mathrm{NO}^{2-}$ was found; with an increase in the growth time, this indicator decreased in proportion to the time.

B: Evaluation of the polymer effect on the biosynthesis of NO in stimulated aorta smooth muscle cells (TAMSCs) [1]

\begin{tabular}{|l|c|c|}
\hline \multirow{2}{*}{ Samples } & \multicolumn{2}{|c|}{ Nitrites production, $4 \%$} \\
\cline { 2 - 3 } & NMMA & \multicolumn{1}{|c|}{} \\
\cline { 2 - 3 } & $0 \mathrm{mM}$ & $1 \mathrm{mM}$ \\
\hline L-Arginine, $500 \mu \mathrm{M}$ & $0 \pm 4.6$ & $-93 \pm 3.6$ \\
\hline Polymer, $50 \mu \mathrm{M}$ & $19 \pm 5.2$ & $-96 \pm 4.2$ \\
\hline Polymer, $500 \mu \mathrm{M}$ & $39 \pm 4.2$ & $-93 \pm 2.5$ \\
\hline R8, $50 \mu \mathrm{M}$ & $17 \pm 5.6$ & $-94 \pm 2.2$ \\
\hline R8, $500 \mu \mathrm{M}$ & $41 \pm 7.0$ & $-90 \pm 4.6$ \\
\hline
\end{tabular}

Note. NO, nitrogen oxide; R8, arginine oligomers; NMMA, NOS inhibitor L-N $\omega$ monomethyl arginine; NOS, nitrogen oxide-synthase.

The use of the polymer as an additional source of substrate for biosynthesis of $\mathrm{NO}$ has increased the production of $\mathrm{NO}$ in activated cells in a dose-dependent manner. The addition of NMMA and inhibitor of NOS, resulted in a significant reduction of nitrites in all relevant samples, indicating that the polymer and arginine oligomers (R8) can increase the production of NO only by providing a substrate to enzymatic reaction. However arginine homo-oligomers have a negative impact on some fundamental metabolic processes [1]

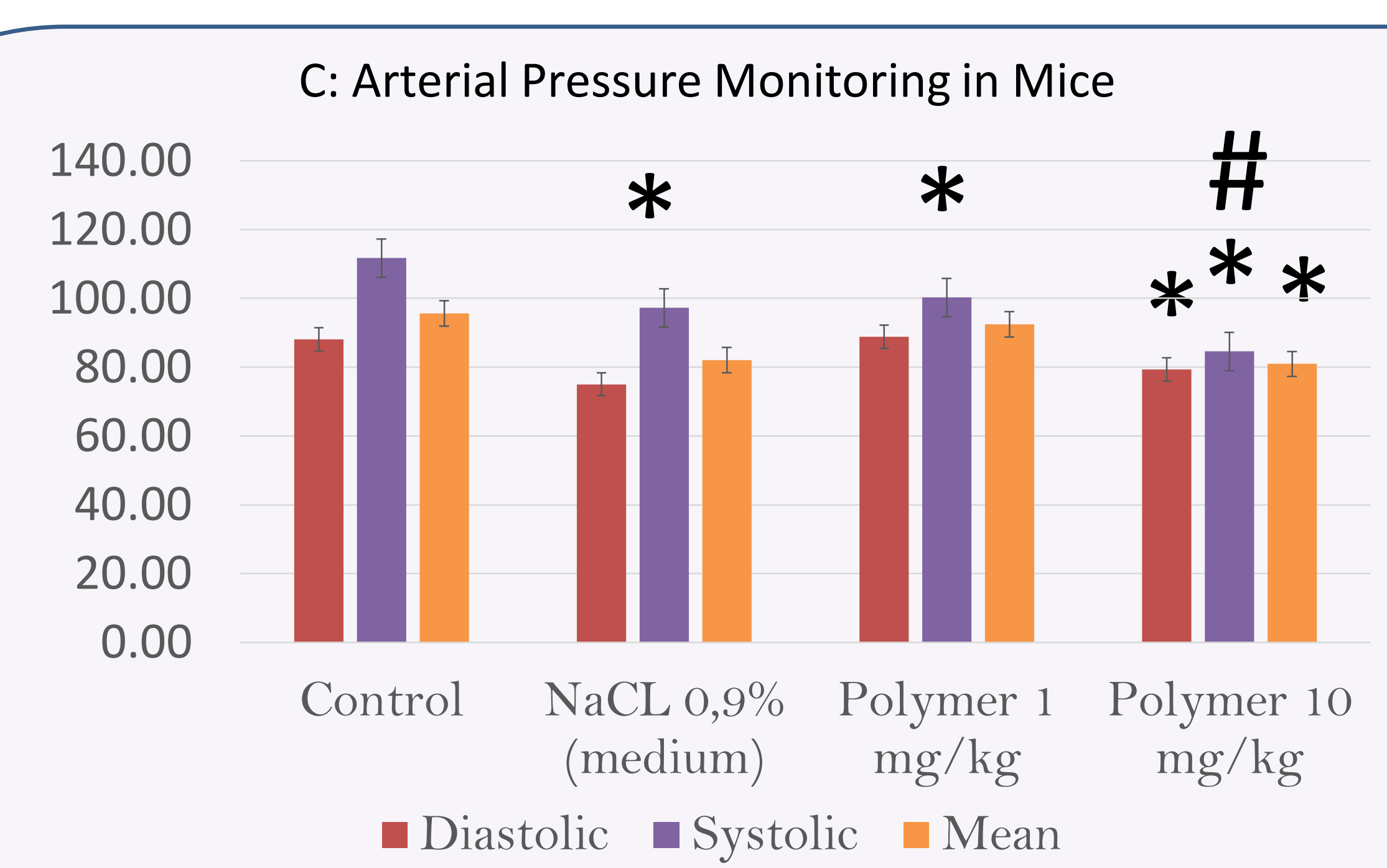

Mean arterial pressure, diastolic and systolic blood pressure in mice CD-1 male Average data are presented as mean \pm standard deviation, statistical analysis was conducted by Mann-Whitney U-test; a shows significant the difference against the control group * $(p<0.05)$ and NACL \# $(p<0.05)$

In vivo study has shown significant decrease in blood pressure compared to intact controls and $\mathrm{NaCl} 0,9 \%$ in a dose-dependent manner. [1] D. Yaroslavtsev, K. Bakhareva, P. Madonov, M. Soldatova, and A. Kotlyarova, "Preparing a Peptide Polymer Structures on Example of L-arginine Grafted Peptide-like Biodegradable Polymer," in 2019 International Multi-Conference on Engineering, Computer and Information Sciences (SIBIRCON), 2019, pp. 0315-0317, do 10.1109/SIBIRCON48586.2019.8958155 